\title{
Sound levels in movie theaters: is there a potential for hearing loss?
}

\begin{abstract}
Purpose: The goal of this study was to determine the frequency of attendance at movie theaters, the most popular genres of movies, the sound levels inside movie theaters, and if, based on frequency of attendance, a relationship could be established between temporary threshold shift and noise levels inside movie theaters.

Method: A survey was first distributed to moviegoers at several different venues. Movies were selected based on the most popular genres. A noise logging dosimeter was utilized during the viewing of 16 movies in two different multiplex theaters to determine if sound levels exceed those deemed hazardous by the Occupational Safety and Health Administration.

Results: Results indicated that most surveyants go to the movie theater about once a week. The noise levels obtained for 16 movies did not exceed those established by OSHA as hazardous in nature.

Conclusion: Given that the maximum dose recorded by any movie viewed was $7.6 \%$ (out of $100 \%$ ), it is highly unlikely that the average person would sustain a temporary threshold shift from movie viewing alone. Even if a person attended the movie theater on a daily basis, there is no evidence to suggest that movie viewing alone could cause a temporary shift in hearing.
\end{abstract}

Keywords: sound levels, hearing loss, non-auditory, physiologic effects, occupational noise

\section{Introduction}

The Occupational Safety and Health Administration (OSHA) have regulated occupationally-based noise exposures in an effort to limit an injury known as noise induced hearing loss (NIHL). Noise induced hearing loss (NIHL) is a disorder characterized by slow-developing hearing loss. Prolonged exposure to high levels of noise can cause permanent damage to hair cells in the cochlea, resulting in varying degrees of noise-induced sensorineural hearing loss. Additionally, noise levels that is intense enough to cause NIHL can also cause non-auditory physiologic effects including stress, inattention, sleep disturbances and elevated blood pressure. ${ }^{1}$

In the United States alone, an estimated 5.2million children and adolescents (ages 6-19) and 26million adults (aged 20-69) have hearing loss caused by exposure to excessively loud noise. ${ }^{2}$ According to the World Health Organization, ${ }^{3}$ only diabetes and cancer are more prevalent, making NIHL the third most chronic health-related condition. The World Health Organization also indicates that almost $50 \%$ of individuals between the ages of 12-35 are exposed to excessive levels of noise from personal audio devices, and that of those, another $40 \%$ might be exposed to harmful sound levels at recreational venues. Currently, there is no effective therapy available to reduce or reverse sensorineural hearing loss (SNHL). Because NIHL is painless, most individuals do not experience any signs or symptoms until it is too late. For this reason, prevention is judicious.

The National Institute for Occupational Safety and Health (NIOSH), the Occupational Safety and Health Administration (OSHA), the European Parliament and Council of the European Union (EPCEU) and other countries including Australia, China, India and Israel have established damage risk criteria (DRC) for NIHL. ${ }^{4} \mathrm{DRC}$ is the permissible exposure level in decibels (PEL), or in terms of a daily noise dose expressed in a percentage based on the regulations for each
Volume II Issue 2 - 2019

\author{
Donna Pitts L \\ Department of Speech-Language-Hearing Sciences, Loyola \\ University Maryland, USA
}

Correspondence: Donna Pitts L, Department of SpeechLanguage-Hearing Sciences, Loyola University Maryland Baltimore, MD 21210, USA, Email Dpitts I@loyola.edu

Received: December 17, 2018 | Published: March 28, 2019 individual country. PELs typically range from 80-90 dB A for an average 8-hour workday. This equates to a dose calculated on a daily basis by combining exposure level with time exposed. A maximum dose for any day is $100 \% .{ }^{5}$ Long-term exposures to levels at or around the PEL results in permanent irreversible damage to the organ of corti, the end organ of hearing in the cochlea. ${ }^{6}$

The focus of most research on this disorder has primarily been on adults exposed to excessive noise in the workplace, where regulations are in place to restrict employees from exposure to intensity, duration and sources of occupational noise. These regulations have been in effect for over 30years. Despite these regulations, some studies have recently indicated that there continues to be an increase in NIHL worldwide over the past 15years, especially in adolescents and young adults. $^{7-11}$ The individuals for whom the regulations were intended to protect (adult workers exposed to occupational noise) are, for the most part, benefiting from the protection these regulations afford; however, regulation and education regarding the likelihood of future NIHL due to recreational activities is deficient. In fact, the risk for NIHL from social causes of noise is increasing in young people. ${ }^{3}$ Recently; some attention has focused on recreationally-based exposures, particularly from activities that are seemingly innocuous. Some of these leisure activities include using personal listening devices, attending concerts and/or nightclubs, attending sporting events such as NASCAR, NHL and NFL games, and attending movies. Several studies have indicated that participating in leisure time activities can further the risk of-or even cause-noise-induced hearing loss. ${ }^{7,12}$

For some people, the acoustics and overall sound effects in movie theaters are part of the overall theater experience because the surround sound experience is difficult to reproduce at home. In light of the recent advances in sound effects at the movie theaters, this investigation evaluated the frequency of attendance at movie theaters, and the potential for unsafe noise levels inside movie theaters during movie 
viewing that might cause temporary threshold shirt (TTS). Only one other study has examined these effects, and the results from that study warranted further investigation. While some recreational venues have been investigated and identified as being hazardous to hearing, only a few studies have considered the sound levels inside movie theaters to determine if these venues can be included in the list of hazardous recreational sites. ${ }^{12-14}$ In 1999, the National Association of Theatre Owners introduced the TASA (Trailer Audio Standards Association) standard, a voluntary certification to limit sound pressure to 85Leq. In order to set this level, movie theaters use a calibrated test signal from the cinema processor, which is $85 \mathrm{dBA} .{ }^{15}$ However, there are no regulations for movie theaters (or film makers for that matter) to make sure the movies are actually played at that calibrated level. In 2014, Connecticut became the first state to consider a proposal that would establish and regulate a maximum decibel level banning movies (and trailers) above $85 \mathrm{dBA}$ (CT General Assembly Bill 287).

In the most recent study, ${ }_{13}^{13}$ utilized calibrated smart phones installed with commercially available software applications to measure sound levels in various locations during the showing of two different movies in ten different theaters. They found significant variance of sound levels across theaters as well as a range of about $3.3 \mathrm{~dB}$, depending on the location of the seating position in the theater. None of the measures in any theater or seat location exceeded the occupational standards for noise. In the next study, ${ }^{10}$ measured 15 movie presentations over a period of six years and as part of a larger study of 500 leisure noise measurements in Australia. None of the movies measured in this study exceeded the occupational noise limits. Prior to that, Warszawa $\&$ Sataloff ${ }^{12}$ measured 25 random movies of different genres in one location in one theater. Their results found that 22 of the 25 movies measured surpassed maximum sound levels, thus indicative of a potential hearing hazard. In the earliest study by Ferguson, Davis \& Lovell, ${ }^{14}$ the researchers viewed and measured four different action movies across four different theaters. They found that none of the movies in any of the theaters exceeded the occupational noise limits.

The purpose of this investigation was two-fold. The first objective was to discover the frequency of attendance at movie theaters to determine if there was a risk for prolonged exposure. The second objective was to measure the sound levels inside movie theaters to determine whether sound levels exceeded those established by OSHA guidelines for occupational noise.

\section{Methodology}

Initially, a five-question survey was provided to individuals age 18 and older as they either entered or exited Movie Theater locations in the suburban Baltimore area in order to determine how often people go to movie theaters, to determine possible risk, and to determine the most popular genres. A total of 447 surveys ( 117 male and 330 female respondents- mean age: 31 ; range of ages: 19-86) were collected over a period of 3 weeks. Of those surveyed, about $1 \%$ indicated that movie attendance was more than once a week, while most indicated going about once a week (27\%) (Figure 1). While the sample size for this portion of the study was large $(n=447)$, it may not accurately represent the movie viewing habits of all individuals. Respondents also indicated which movie genre they were most likely to see, and movies were selected from these criteria to measure sound levels (Figure 2).

Movies were viewed over a period of 6weeks in two different multiplex theaters, where each theater was similar in size and seating capacity. All measurements were taken at the center of each theater to ensure equality among measures. Additionally, all measures were made at ear level for application closest to OSHA standards. Movies were selected based on the survey responses of favorite genre. Seven comedies, six actions, one musical and one action/comedy were measured and viewed over a period of approximately six weeks. No assumptions regarding movie rating were made, although three (Hercules, Planes: Fire and Rescue, and How to Train Your Dragon) of the fifteen movies viewed were animated and more likely to target children and adolescent viewers. Further, measurements from each multiplex were compared to determine if there was a difference in levels between theaters.

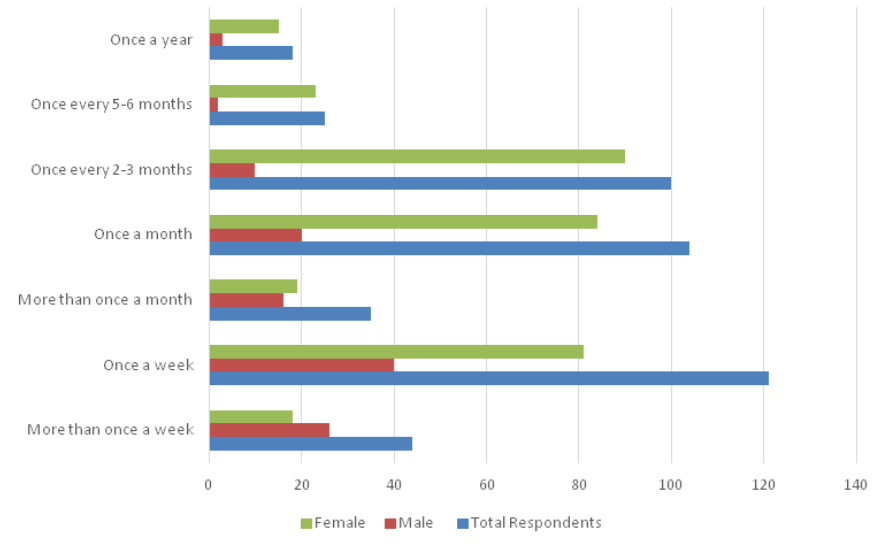

Figure I Movie theater attendance $(\mathrm{N}=447)$.

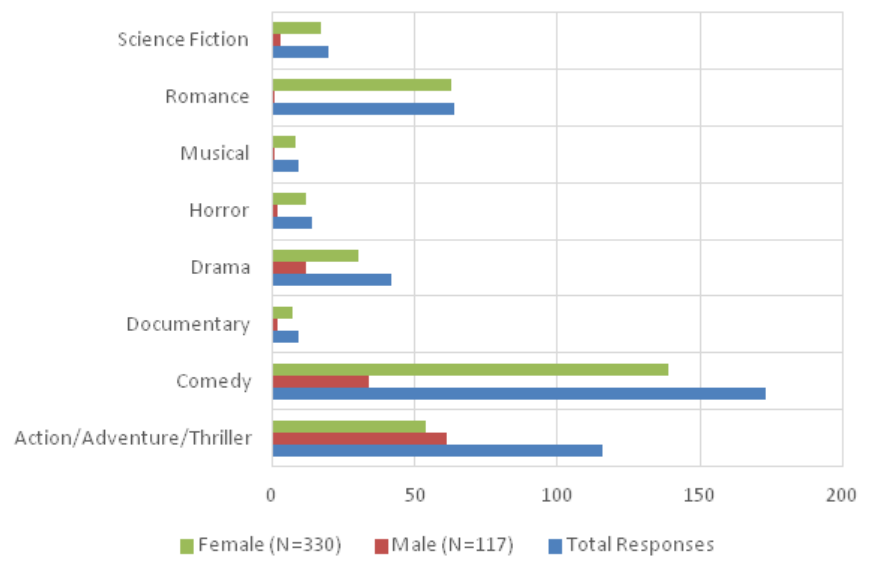

Figure 2 Total surveyants $\mathrm{N}=447$.

Noise levels were measured with a Quest Pro noise logging dosimeter and data was analyzed with the Quest Suite Professional software. ${ }^{15}$ All measurements were made using A-weighting; slow averaging, and a $5 \mathrm{~dB}$ exchange rate. Each movie viewed had the following sound measurements recorded (Table $1 \&$ Table 2):

a. The maximum sound level (the highest sound level measured).

b. Percent dose (where $100 \%$ is maximum and occurs for average sound levels of $90 \mathrm{~dB}$ A over an 8hour period).

c. The average sound level $\left(\mathrm{L}_{\text {avg }}\right)$ (OSHA preferred over $\left.\mathrm{L}_{\mathrm{eq}}\right)$.

\section{Results}

While viewing times ranged from $4: 45 \mathrm{pm}-11 \mathrm{pm}$ on Thursday, Friday and Saturday nights, there were no significant differences 
between the multiplex cinemas size and capacity at either location during the recording of sound levels. All data was collected during summer months with the assumption that movie viewing would be similar throughout the year.

Table I Cumulative running time and percent dose

\begin{tabular}{lll}
\hline Title of movie & $\begin{array}{l}\text { Running time } \\
\text { (minutes) }\end{array}$ & $\begin{array}{l}\text { Percent } \\
\text { Dose (\%) }\end{array}$ \\
\hline Are You Here? & 114 & 2.1 \\
Behaving Badly & 97 & 2.4 \\
Dawn of the Planet of the Apes & 130 & 4.6 \\
Guardians of the Galaxy & 121 & 1.6 \\
Hercules (animated) & 93 & 7.4 \\
How to Train Your Dragon 2 (animated) & 102 & 1.1 \\
Into the Storm (2-D) & 89 & 7.6 \\
Jersey Boys & 134 & 7.5 \\
Lucy & 89 & 3.3 \\
Magic in the Moonlight & 97 & 0.6 \\
Mom's Night Out & 98 & 0.2 \\
Planes Fire and Rescue & 83 & 2 \\
Tammy & 97 & 0.2 \\
Teenage Mutant Ninja Turtles & 101 & 3.8 \\
Transformers 4:Age of Extinction & 165 & 6.5 \\
\hline
\end{tabular}

Maximum sound level: The highest maximum sound levels were recorded at Transformers 4: Age of Extinction (133.8dB A-Multiplex A), Dawn of the Planet of the Apes (131.9dB A-Multiplex B), and Jersey Boys (128.0dB A- Multiplex A) (Figure 3). One-Way Analysis of Variance (ANOVA) between groups (Multiplex A and Multiplex B) was calculated. No statistical differences were found in maximum sound levels between recordings for any movie at either multiplex theater, so only the highest maximum sound levels for individual films are listed. Two of the movies exceeded the ceiling limits instituted by OSHA, each for less than a second.

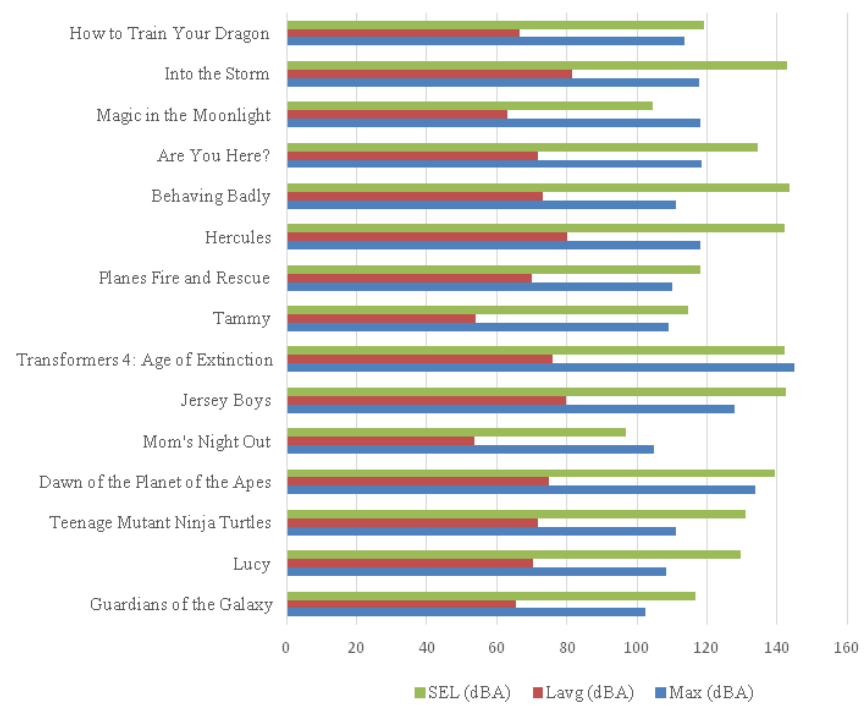

Figure 3 Measured sound levels.

Table 2 Cumulative time of exposure to different sound exposure levels

\begin{tabular}{|c|c|c|c|c|c|c|}
\hline Title of movie & Running time & $\geq 90 \mathrm{dBA}$ & $\geq 100 \mathrm{dBA}$ & $\geq I I 0 d B A$ & $\geq 120 \mathrm{dBA}$ & $\geq 130 \mathrm{dBA}$ \\
\hline Guardians of the Galaxy & 121 & 39 & 2 & - & - & - \\
\hline Lucy & 89 & 40 & 9 & $3 \mathrm{sec}$ & & \\
\hline Teenage Mutant Ninja Turtles & 101 & & & & & \\
\hline Dawn of the Planet of the Apes & 130 & & & & & \\
\hline Mom's Night Out & 98 & & & & & \\
\hline Jersey Boys & 134 & & & & & \\
\hline Transformers 4:Age of Extinction & 165 & & & & & \\
\hline Tammy & 97 & & & & & \\
\hline Planes: Fire and Rescue (animated) & 83 & & & & & \\
\hline Hercules (animated) & 93 & & & & & \\
\hline Behaving Badly & 97 & & & & & \\
\hline Are you Here? & 114 & & & & & \\
\hline Magic in the Moonlight & 97 & & & & & \\
\hline Into the Storm (2-D) & 89 & & & & & \\
\hline How to Train Your Dragon 2 (animated) & 102 & & & & & \\
\hline
\end{tabular}


Percent dose: Percent dose was analyzed for each movie based on the OSHA criteria. Longer duration movies would be expected to have a higher dose calculation. The highest percent dose was calculated for the following movies: Into the Storm (7.6\%; 89minutesMultiplex B), Hercules (7.5 \%; 93 minutes-Multiplex A), and Jersey Boys $(7.5 \%$; 134minutes-Multiplex A). Three of the movies sampled had doses below one percent: Mom's Night Out and Tammy (both 0.2 $\%$ at Multiplex A) and Magic in the Moonlight (0.6\%-Multiplex B). One-Way Analysis of Variance (ANOVA) between groups (Multiplex A and Multiplex B) was calculated. No statistical differences were found in percent dose between recordings for any movie at either multiplex, so only the highest dose is listed.

$\mathbf{L}_{\text {avg }}$ : The highest $\mathrm{L}_{\mathrm{AVG}}$ was recorded for the following movies: Into the Storm (81.9dB A-Multiplex B), Hercules (80.1dB A-Multiplex A) and Jersey Boys (79.9dB A-Multiplex A) (Table 1). Most concerning was the $80.1 \mathrm{dBA}$ from Hercules, which is an animated movie geared more towards children. The lowest recorded $\mathrm{L}_{\mathrm{AVG}}$ were Mom's Night Out (53.5dB A-Multiplex A), Tammy (54.1dB A-Multiplex A), and Magic in the Moonlight (63.0dB A-Multiplex B). One-Way Analysis of Variance (ANOVA) between groups (Multiplex A and Multiplex B) was calculated. No statistical differences were found in $L_{\text {avg }}$ between recordings for any movie at either multiplex, so only the highest $L_{\text {avg }}$ is listed.

\section{Discussion}

There are no current guidelines for non-occupational noise induced hearing loss, even though studies across the globe have shown an increase in the disorder in recent history, and particularly in young adults. This drastic increase implies that behavioral and informational actions have changed over the past 15 years. Awareness and knowledge can influence attitudes and beliefs towards noise. Few studies have attempted to demonstrate the relationship between sound levels inside movie theaters and the potential for non-occupational noise induced hearing loss. According to information collected from NATO and TASA, it seems the movie industry is aware of the potential for hazardous sound levels and is making attempts to standardize sound levels for films. This was apparent, as there were no statistical differences across the two multiplex theaters. Also, newer digital technology used in movie production appears to enhance the overall quality of the sound while attempting to eliminate noise. ${ }^{16-18}$

In this study, none of the fifteen movies viewed exceeded the OSHA ceiling (140dBA) for noise exposure levels, and the overall dose for each of the movies was well under the daily noise dose established for occupational exposure. Given that the maximum dose recorded by any movie viewed for this research was $7.6 \%$, it is highly unlikely that the average person who views movies on a weekly basis would sustain a temporary threshold shift from movie viewing alone. Even if a person attended the movie theater on a daily basis, there is no evidence to suggest that movie viewing alone could cause a temporary shift in hearing. Further, 15movies represented approximately 25hours (25.67), while the dose accumulated over that time period was $50.9 \%$. Even if a person watched movies continuously over a 24 hour period, they would not exceed the daily dose for occupational noise exposure. While the results of this study may be generalized to the adult population based on OSHA standards (2008), no standards exist for children. It is unknown if children's' hearing is more susceptible to lesser noise than in adults. Precautions should be taken for overzealous movie viewing in young children until such standards are in place.

\section{Acknowledgments}

None.

\section{Conflicts of interest}

Author declares that there are no conflicts of interest.

\section{References}

1. Dell SM, Homes AE. The effect of a hearing conservation program on adolescent's attitudes towards noise. Noise Health. 2012;14(56):39-44.

2. Centers for Disease Control and Prevention (CDC). Adolescent and School Health: Noise-Induced Hearing Loss. Atlanta, GA; 2017.

3. World Health Organization (WHO). Fact Sheet on Deafness and Hearing Loss. 2017.

4. Crocker M. Handbook of Noise and Vibration Control. Wiley and Sons: Hoboken, New Jersey; 2007.

5. OSHA. Occupational safety and health standards. In 1910.95 Occupational Noise Exposure. Washington DC: Occupational Safety and Health Administration; 2008.

6. Sliwinski Kowalska M, Davis A. Noise-induced hearing loss. Noise and Health. 2012;14:274-280.

7. Serra MR, Biasonni EC, Richter U, et al. Recreational noise exposure and its effect of the hearing of adolescents. Part 1: An interdisciplinary long-term study. Int J Audiol. 2005;44(2):65-73.

8. Vogel I, Verschuure H, Raat H. Estimating adolescent hearing loss based on data from a large school-based survey. Am J Public Health. 2010;100(6):1095-1102

9. Zhao F, Manchaiah VK, French D. Music exposure and hearing disorders. Int J Audiol. 2010;49(1):54-64.

10. Beach EF, Gilliver M, Williams W. The NOISE (Non-Occupational Incidents,Situations and Events) database: A new research tool. Annals of Leisure Research. 2013;16(2):149-159.

11. Pitts DL. Noise induced hearing loss in youth: An international concern. Perspectives on Global Issues in Communication Sciences and Related Disorders. 2013;3(2):68-72.

12. Warszawa A, Sataloff RT. Noise exposure in movie theaters: A preliminary study of sound levels during the showing of 25 films. Ear Nose Throat J. 2010;89(9):444-450.

13. Huth ME, Popelka GR, Blevins NH. Comprehensive measures of sound exposures in cinemas using smart phones. Ear Hear. 2014;35(6):680 686.

14. Ferguson MA, Davis AC, Lovell EA. Cinemas-Do they pose a risk to hearing? Noise Health. 2000;2(8):55-58.

15. Quest Suite Professional. 2014.

16. National Association of Theater Owners. NATO-UNIC. Immersive sound requirements. 2016

17. CT. General Assembly. An act concerning the maximum decibel level in movie theatres. 2014.

18. Trailer Audio Standards Association (TASA). What is TASA? 2014. 